TITLE:

\title{
Ultrasonic wave transmission and bandgap in multidirectional composite laminates with spring- type interlayer interfaces
}

$\operatorname{AUTHOR}(S)$ :

Ishii, Yosuke; Biwa, Shiro

\section{CITATION:}

Ishii, Yosuke ... [et al]. Ultrasonic wave transmission and bandgap in multidirectional composite laminates with spring-type interlayer interfaces. Journal of the Acoustical Society of America 2017, 141: 1099-1110

ISSUE DATE:

2017-02-01

URL:

http://hdl.handle.net/2433/226601

RIGHT:

(C) 2017 Acoustical Society of America. 


\title{
Ultrasonic wave transmission and bandgap in multidirectional composite laminates with spring-type interlayer interfaces
}

\author{
Yosuke Ishii $^{\mathrm{a})}$ and Shiro Biwa \\ Department of Aeronautics and Astronautics, Graduate School of Engineering, Kyoto University, Katsura, \\ Nishikyo-ku, Kyoto 615-8540, Japan
}

(Received 17 June 2016; revised 5 January 2017; accepted 7 January 2017; published online 24 February 2017)

\begin{abstract}
The ultrasonic wave transmission through multidirectional composite laminates is studied theoretically by accounting for the effect of thin interlayer resin-rich regions based on the spring-type interface model. Using the stiffness-matrix method, the energy transmission spectrum of the longitudinal wave impinging obliquely on cross-ply and quasi-isotropic laminates immersed in water is calculated. The location and bandwidth of the frequency ranges where the transmissivity becomes vanishingly small are shown to be significantly influenced by the incident angle, the laminate lay-up, and the interlayer interfacial stiffnesses. By examining the energy flux density of partial waves inside the laminate, these frequency ranges are shown to be the bandgaps due to the constructive interference of scattered waves from the interlayer interfaces. The mode combination causing the interference is found to vary remarkably with the bandgap location. Furthermore, the interference in the finite laminate structure is shown to occur in almost the same manner as the Floquet wave does in the infinitely extended laminate structure. The energy transmission spectrum is experimentally measured for 16-ply carbon/epoxy cross-ply and quasi-isotropic composite laminates using the through-transmission technique. The transmission and bandgap characteristics observed in the experimental results are reasonably reproduced by the present theory incorporating the interlayer resin-rich regions. (C) 2017 Acoustical Society of America.
\end{abstract}

[http://dx.doi.org/10.1121/1.4976062]

[AGP]

Pages: 1099-1110

\section{INTRODUCTION}

In multilayered structures with a stacking periodicity, the wave transmission is largely prohibited in certain frequency ranges due to the constructive interference of scattered waves from the interlayer interfaces. These frequency ranges are called bandgaps or stop-bands. The occurrence of bandgaps has been extensively studied for multilayered structures consisting of isotropic media ${ }^{1-14}$ for the purpose of fabricating artificial crystals called superlattices, ${ }^{15,16}$ designing foundations to isolate buildings from seismic waves, ${ }^{17,18}$ and so on. Meanwhile, the advent of advanced fiber-reinforced composite materials in the mid-20th century motivated researchers to investigate the bandgap formation of multilayered anisotropic media. ${ }^{19-24}$ Among others, Braga and Herrmann ${ }^{25}$ formulated the characteristic equation of Floquet waves propagating in infinitely stratified anisotropic layers by combining the Stroh formalism ${ }^{26}$ with the Floquet theorem. They elucidated the band structure, namely, the dispersion relation of Floquet waves, of an infinite structure with cross-ply layering. The propagation characteristics of Floquet waves were also investigated for the case of quasi-isotropic layering by Potel et al. ${ }^{27}$ and Wang and Rokhlin. ${ }^{28}$ The revealed bandgap behavior was utilized to determine the validity domain (frequency

\footnotetext{
${ }^{a)}$ Current address: Department of Mechanical Engineering, Toyohashi University of Technology, 1-1 Hibarigaoka, Tempaku-cho, Toyohashi 441-8580, Japan. Electronic mail: ishii@me.tut.ac.jp
}

and incident direction) of the so-called Floquet wave homogenization. ${ }^{27-31}$

The above-mentioned works ${ }^{1-31}$ assumed in common that the neighboring layers of multilayered structures were perfectly bonded, i.e., the displacements as well as stresses are continuous across the interlayer interfaces. In actual carbon/epoxy and glass/epoxy composite laminates, however, thin resin-rich regions with typically several microns thickness usually exist between adjacent plies. Understanding the influence of such interfacial regions on the ultrasonic wave propagation is essential for nondestructive diagnosis of the interface quality, which has significant effects on the mechanical performance of the whole laminated structure. ${ }^{32}$ Wang and Rokhlin ${ }^{28}$ modeled the interlayer resin-rich regions of composite laminates by thin elastic layers of finite thickness and discussed the bandgap behavior at normal incidence. According to Rokhlin and Wang, ${ }^{33}$ such thin interface layers can also be modeled as spring-type interfaces ${ }^{34-43}$ with equivalent stiffnesses and neglected mass. Using this model, $\mathrm{Lu}$ and Achenbach ${ }^{44}$ and $\mathrm{Lu}^{45}$ analyzed the influence of random fluctuations of the interfacial normal stiffness ${ }^{44}$ or the wave velocity in layers ${ }^{45}$ on the wave reflection/ transmission characteristics of longitudinal wave at normal incidence. Ishii and Biwa ${ }^{46-48}$ elucidated the influence of the interlayer interfacial stiffnesses on the wave propagation behavior, including the bandgap formation in the layering direction ${ }^{46,47}$ as well as arbitrary directions in unidirectional composite laminates ${ }^{48}$ with spring-type interlayer interfaces. These foregoing studies ${ }^{28,44-48}$ account for the presence of 
interlayer resin-rich regions, but are limited to the case of normal incidence to multidirectional laminates or oblique incidence to unidirectional laminates where the interlayer interfaces are solely responsible for the bandgap formation. In the more general case of oblique incidence to multidirectional composite laminates, the wave scattering occurs not only due to the finite interfacial stiffnesses but also due to the mismatch of acoustic impedances between neighboring plies, so the resulting bandgap formation remains as an issue needing in-depth investigation.

In the present study, the ultrasonic wave transmission characteristics and the bandgap formation in multidirectional composite laminates with spring-type interlayer interfaces are investigated theoretically. In particular, the influence of the angle of incidence, the stacking sequence of laminates, and the interlayer interfacial stiffnesses on the energy transmission spectrum is elucidated. In Sec. II, the stiffness-matrix method ${ }^{49,50}$ is employed to calculate the energy transmission coefficient of the longitudinal wave at oblique incidence to a composite laminate immersed in water. The resulting transmission spectra of cross-ply and quasi-isotropic composite laminates are presented and discussed in Sec. III. In order to discuss the formation mechanism of low-transmission frequency ranges found in the spectrum in more detail, the energy flux density of partial waves inside the laminate is examined in Sec. IV A. The energy flux of partial waves constituting the Floquet waves as well as the number of propagative Floquet modes in the infinitely extended laminate structure are also calculated and compared to the finite layered case in Sec. IV B. The energy transmission spectrum is experimentally measured for 16-ply carbon/epoxy composite laminates with cross-ply and quasi-isotropic stacking sequences and compared to the theoretical results in Sec. V. The conclusion of the present study is summarized in Sec. VI.

\section{ANALYSIS OF WAVE TRANSMISSION CHARACTERISTICS}

This study deals with the transmission of plane harmonic longitudinal wave impinging obliquely upon a composite laminate immersed in water (density $\rho_{\mathrm{f}}$ and wave speed $V_{\mathrm{f}}$ ) as shown in Fig. 1. Specifically, two types of stacking sequences are considered for the composite laminate: a symmetric cross-ply $[0 / 90]_{4 \mathrm{~S}}$ and a symmetric quasiisotropic $[+45 / 0 /-45 / 90]_{2 S}$ (Fig. 1) lay-up, both of which consist of 16 transversely isotropic elastic plies and 15 spring-type interlayer interfaces. The procedure of the analysis is, however, outlined in this section for the laminate structure made of $N$ layers with $N-1$ spring-type interlayer interfaces. It is noted here that interlayer resin-rich regions of composite laminates are sufficiently thin compared to the ultrasonic wavelength in the frequency range of $0-14 \mathrm{MHz}$ considered in the present analysis: for example, the carbon/ epoxy cross-ply composite laminate, which will be used in the experiment in Sec. $\mathrm{V}$, has the resin-rich regions of typically 3-8 $\mu \mathrm{m}$ thickness, ${ }^{46}$ while the wavelengths of the longitudinal and shear waves in the same epoxy resin as the composite laminate $^{51}$ are about $0.2 \mathrm{~mm}$ and $0.09 \mathrm{~mm}$ at

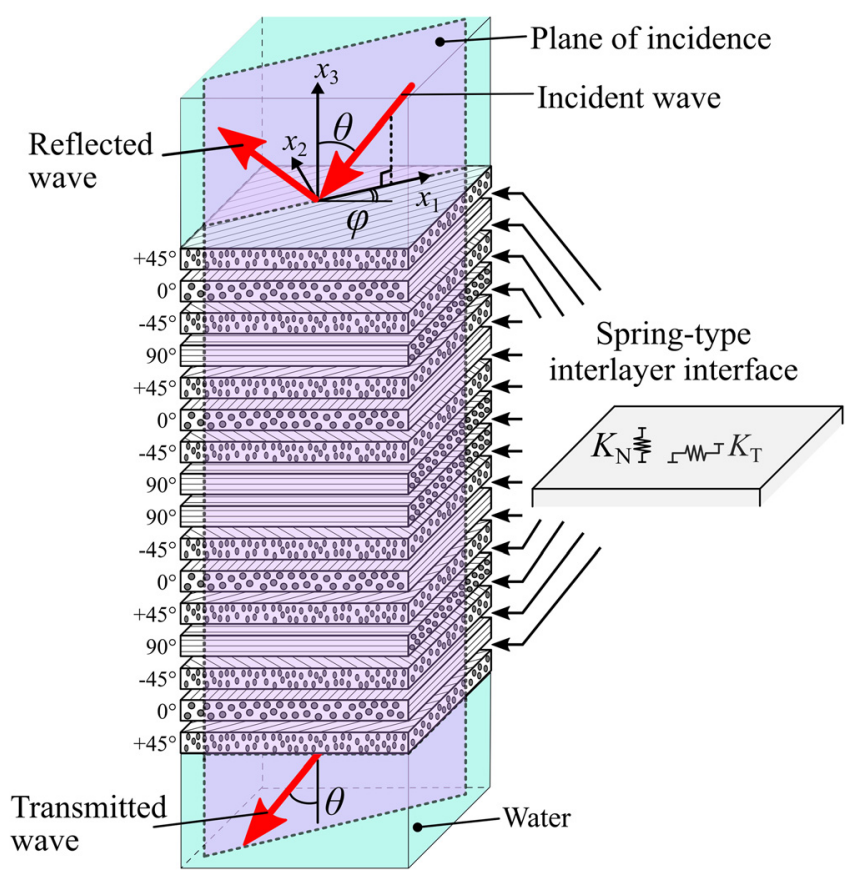

FIG. 1. (Color online) A 16-ply quasi-isotropic composite laminate with spring-type interlayer interfaces immersed in water.

$14 \mathrm{MHz}$, respectively. Therefore, the spring-type interface model can be reasonably applied to model the thin resinrich regions.

The Cartesian coordinate system is set in such a manner that the $x_{3}$ axis coincides with the stacking direction and the $x_{1}-x_{3}$ plane with the plane of incidence as shown in Fig. 1. The angle $\varphi$ is defined as the deviation of the plane of incidence from the isotropic plane of the $0^{\circ}$ plies.

When the displacement vector is defined as $\mathbf{u} \equiv\left(u_{1}, u_{2}, u_{3}\right)^{\mathrm{T}}$, where the superscript " $\mathrm{T}$ " denotes the transpose, the displacement fields of the incident and the resulting transmitted longitudinal waves are given as

$$
\begin{aligned}
\mathbf{u}^{\text {Inc }}= & A_{\text {inc }}\left(\begin{array}{c}
\sin \theta \\
0 \\
\cos \theta
\end{array}\right) e^{-i\left\{k_{\mathrm{f}}\left[x_{1} \sin \theta+\left(x_{3}-Z_{0}\right) \cos \theta\right]+\omega t\right\}}, \quad x_{3}>Z_{0} \\
\mathbf{u}^{\text {Tra }}= & T A_{\text {inc }}\left(\begin{array}{c}
\sin \theta \\
0 \\
\cos \theta
\end{array}\right) e^{-i\left\{k_{\mathrm{f}}\left[x_{1} \sin \theta+\left(x_{3}-Z_{0}\right) \cos \theta\right]+\omega t\right\}} \\
& x_{3}<Z_{N}
\end{aligned}
$$

where $A_{\text {inc }}$ is the amplitude of incident wave, $\theta$ is the angle of incidence, $i^{2}=-1, k_{\mathrm{f}}=\omega / V_{\mathrm{f}}$ is the wavenumber in water, $\omega$ is the angular frequency, $t$ is the time, and $x_{3}=Z_{0}$ and $x_{3}=Z_{N}$ are the positions of top and bottom surfaces of the laminate, respectively. In Eq. (2), $T$ denotes the complex amplitude transmission coefficient. This can be calculated by using the stiffness-matrix method ${ }^{49,50}$ as

$$
T=\frac{-2 \gamma S_{63}^{\mathrm{G}}}{\left(\gamma-S_{33}^{\mathrm{G}}\right)\left(\gamma+S_{66}^{\mathrm{G}}\right)+S_{36}^{\mathrm{G}} S_{63}^{\mathrm{G}}} e^{-i k_{\mathrm{f}} H \cos \theta},
$$


where $\gamma \equiv \cos \theta /\left(i \rho_{\mathrm{f}} V_{\mathrm{f}} \omega\right)$ and $H=Z_{0}-Z_{N}$ is the laminate thickness. In Eq. (3), $S_{I J}^{\mathrm{G}}$ represent the elements of the inverse of $6 \times 6$ global stiffness matrix, which is obtained by combing the local stiffness matrices of the plies $\mathbf{K}_{m}^{\text {ply }}(m=1$, $2, \ldots, N)$ and the interlayer interfaces $\mathbf{K}_{p}^{\mathrm{sp}}(p=1,2, \ldots, N-1)$ using the recursive algorithm. ${ }^{49}$ These local matrices are defined as

$$
\begin{aligned}
& \left(\begin{array}{c}
\left.\boldsymbol{\sigma}\right|_{x_{3}=Z_{m-1,-}} \\
\left.\boldsymbol{\sigma}\right|_{x_{3}=Z_{m,+}}
\end{array}\right)=\mathbf{K}_{m}^{\mathrm{ply}}\left(\begin{array}{c}
\left.\mathbf{u}\right|_{x_{3}=Z_{m-1,-}} \\
\left.\mathbf{u}\right|_{x_{3}=Z_{m,+}}
\end{array}\right), \\
& \left(\begin{array}{c}
\left.\boldsymbol{\sigma}\right|_{x_{3}=Z_{p,+}} \\
\left.\boldsymbol{\sigma}\right|_{x_{3}=Z_{p,-}}
\end{array}\right)=\mathbf{K}_{p}^{\mathrm{sp}}\left(\begin{array}{c}
\left.\mathbf{u}\right|_{x_{3}=Z_{p,+}} \\
\left.\mathbf{u}\right|_{x_{3}=Z_{p,-}}
\end{array}\right),
\end{aligned}
$$

where $\boldsymbol{\sigma} \equiv\left(\sigma_{13}, \sigma_{23}, \sigma_{33}\right)^{\mathrm{T}}$ is the stress vector, $x_{3}=Z_{p}$ $(p=1,2, \ldots, N-1)$ are the positions of interlayer interfaces, and the subscripts "+" and "-" denote the limit of a field variable when $x_{3}$ approaches the corresponding coordinate from the positive and negative sides, respectively. The local stiffness matrix of the ply can be calculated according to the procedure in Ref. 49, while that of the interlayer interface is given as

$$
\mathbf{K}_{p}^{\mathrm{sp}}=\left[\begin{array}{ll}
\mathbf{B}_{p} & -\mathbf{B}_{p} \\
\mathbf{B}_{p} & -\mathbf{B}_{p}
\end{array}\right], \quad \mathbf{B}_{p}=\left[\begin{array}{ccc}
K_{\mathrm{T} 11} & \mathrm{~K}_{\mathrm{T} 12} & 0 \\
K_{\mathrm{T} 21} & K_{\mathrm{T} 22} & 0 \\
0 & 0 & K_{\mathrm{N}}
\end{array}\right],
$$

where $K_{\mathrm{N}}$ is the interfacial normal stiffness, and $K_{\mathrm{T} 11}$, $K_{\mathrm{T} 12}, K_{\mathrm{T} 21}$, and $K_{\mathrm{T} 22}$ are terms governed by the shear interfacial stiffness, which generally depends on the fiber direction of plies on both sides of the interface and the angle $\varphi$.

In the present analysis, all plies are assumed to have the same material properties of density $\rho$, thickness $h$, and elastic constants with respect to their crystallographic coordinates in Voigt notation $C_{i j}$. Namely, the stiffness matrix of the $m$ th ply $\mathbf{K}_{m}^{\text {ply }}$ depends on $m$ due only to its fiber direction. In addition, it is assumed that all interlayer interfaces possess the same normal and orientation-independent shear stiffnesses $K_{\mathrm{N}}$ and $K_{\mathrm{T}}$, and that the shear stresses occur at the interlayer interfaces only in the direction of the shear displacement gap. In this case, $\mathbf{B}_{p}$ in Eq. (5) becomes $\mathbf{B}_{p}$ $=\operatorname{diag}\left(K_{\mathrm{T}}, K_{\mathrm{T}}, K_{\mathrm{N}}\right)$ for any stacking sequences and any angles $\varphi$.

\section{RESULTS}

\section{A. Composite laminates with perfectly bonded interlayer interfaces}

The numerical results are first shown for the case of perfect bonding at interlayer interfaces, i.e., $K_{\mathrm{N}}=K_{\mathrm{T}}=\infty$, for a better understanding of the case of finite interfacial stiffnesses, which will be presented in Sec. III B. It is noted that the wave transmission characteristics of multidirectional composite laminates with perfectly bonded interfaces have been studied by Wang and Rokhlin. ${ }^{28}$

The variation of the energy transmission coefficient $|T|^{2}$ calculated by Eq. (3) with the frequency $f$ and the angle

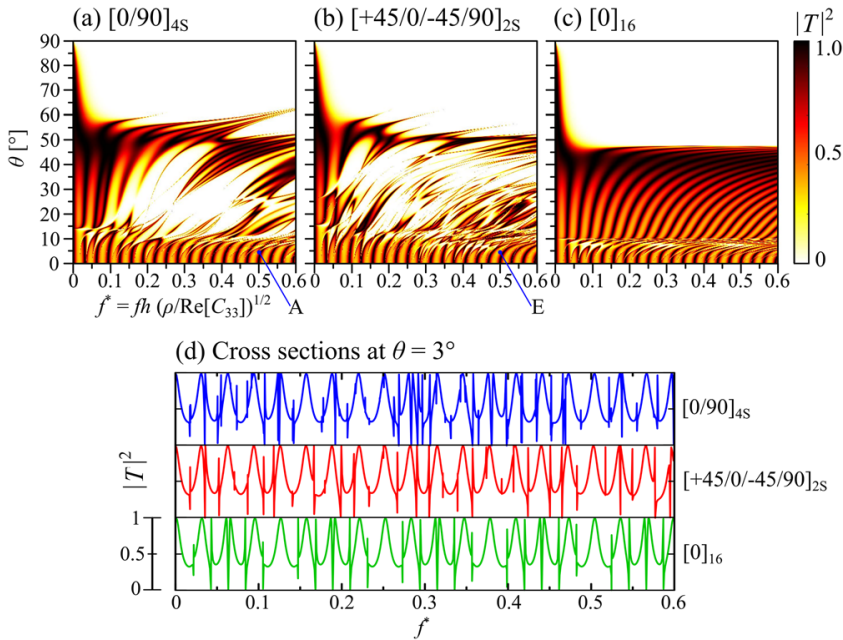

FIG. 2. (Color online) Variation of the energy transmission spectrum of 16ply (a) cross-ply, (b) quasi-isotropic, and (c) unidirectional composite laminates with the angle of incidence $\theta$ when $\varphi=90^{\circ}$ and $K_{\mathrm{N}}=K_{\mathrm{T}}=\infty$, and (d) the cross sections of (a)-(c) at $\theta=3^{\circ}$.

of incidence is shown in Figs. 2(a) and 2(b) for the crossply and quasi-isotropic laminates, respectively. The results for a 16-ply unidirectional laminate $[0]_{16}$ are also shown for comparison in Fig. 2(c). The material properties used in the present study are summarized in Table I. Note that the imaginary parts of ply elastic constants in Table I are neglected in Secs. III and IV, but will be considered in the comparison of the analysis with experiments in Sec. V. The angle of the plane of incidence is fixed as $\varphi=90^{\circ}$. The horizontal axis $f^{*} \equiv f h\left(\rho / \operatorname{Re}\left[C_{33}\right]\right)^{1 / 2}$ in Fig. 2 represents the frequency normalized by the ply thickness and the longitudinal wave velocity of the ply in the $x_{3}$ direction, whose range corresponds approximately to $0 \leq f \leq 14 \mathrm{MHz}$.

It is seen in Figs. 2(a) and 2(b) that when approximately $\theta<10^{\circ}$, both cross-ply and quasi-isotropic laminates exhibit the similar oscillatory pattern of the transmission coefficient against the frequency to that of the unidirectional lay-up in Fig. 2(c). As an example, cross sections of Figs. 2(a)-2(c) at a fixed incident angle of $\theta=3^{\circ}$ are shown in Fig. 2(d). Except for the several sharp peaks due to the occurrence of eigen vibration of the laminates, the spectra in Fig. 2(d) have very similar oscillation amplitude and period irrespective of the stacking sequence. On the other hand, the transmission characteristics in the range of approximately $10^{\circ}<\theta<60^{\circ}$

TABLE I. Material properties of the ply and the water.

Complex elastic constants of transversely

isotropic ply (GPa; fiber direction: $x_{1}$ )

$C_{11}$

$109-2.90 i$

$C_{13}$

$4.9-0.18 i$

$C_{33}$

$14.8-0.33 i$

$C_{44}$

$C_{66}$

$3.6-0.16 i$

Density of ply $\rho\left(\mathrm{kg} / \mathrm{m}^{3}\right)$

$6.2-0.30 i$

Thickness of ply $h(\mathrm{~mm})$

$1.5 \times 10^{3}$

Density of water $\rho_{\mathrm{f}}\left(\mathrm{kg} / \mathrm{m}^{3}\right)$

0.135

$1.0 \times 10^{3}$

Wave speed in water $V_{\mathrm{f}}(\mathrm{m} / \mathrm{s})$ 
are significantly influenced by the stacking sequence. Unlike the unidirectional lay-up, the cross-ply and quasi-isotropic laminates have several finite frequency ranges of low transmissivity in a manner depending on the angle of incidence, such as the one at around $f^{*}=0.3$ when $\theta=40^{\circ}$ in Fig. 2(a). The occurrence of such low-transmission zones will be demonstrated in the case of laminates with spring-type interlayer interfaces below, and discussed further in Sec. IV in the light of bandgaps of Floquet waves in the corresponding infinite periodic structures. When the angle of incidence is sufficiently large $\left(\theta>60^{\circ}\right)$, the transmission coefficient in Figs. 2(a)-2(c) becomes very small over the wide frequency ranges because of the total reflection of the incident wave at the water-laminate interface.

\section{B. Composite laminates with spring-type interlayer interfaces}

When the thin interlayer resin-rich zones of composite laminates are modeled as spring-type interfaces, the interfacial normal and shear stiffnesses can be reasonably approximated as $K_{\mathrm{N}}=\left(\lambda_{\mathrm{e}}+2 \mu_{\mathrm{e}}\right) / h_{\mathrm{e}}$ and $K_{\mathrm{T}}=\mu_{\mathrm{e}} / h_{\mathrm{e}}{ }^{33}$ respectively, where $\lambda_{\mathrm{e}}$ and $\mu_{\mathrm{e}}$ are the Lamé constants of resin and $h_{\mathrm{e}}$ is the equivalent thickness of resin-rich zones. The two interfacial stiffnesses are hence expected to have a certain correlation for actual composite laminates, i.e., $K_{\mathrm{N}} / K_{\mathrm{T}}=\left(\lambda_{\mathrm{e}}+2 \mu_{\mathrm{e}}\right) / \mu_{\mathrm{e}}$. In this section, however, the influence of each of normal and shear stiffnesses on the wave transmission characteristics as well as bandgap behavior is investigated separately.

In order to first examine the influence of interfacial normal stiffness $K_{\mathrm{N}}$, the interfacial shear stiffness is fixed as $K_{\mathrm{T}}=\infty$ and the variation of the energy transmission spectrum of the cross-ply and quasi-isotropic laminates with $K_{\mathrm{N}}$ is depicted in Fig. 3 for two representative angles of incidence $\theta=5^{\circ}$ and $40^{\circ}$ for small $\left(\theta<10^{\circ}\right)$ and large

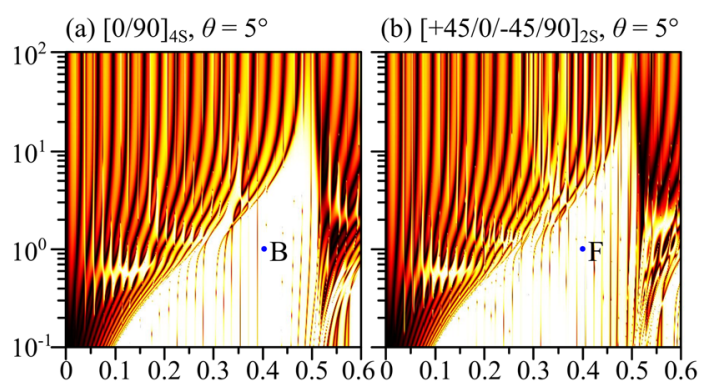

(c) $[0 / 90]_{4 \mathrm{~S}}, \theta=40^{\circ}$

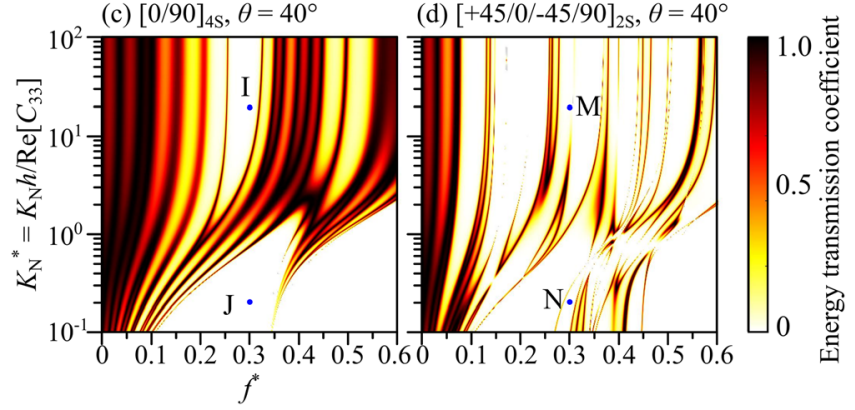

FIG. 3. (Color online) Dependence of the energy transmission spectrum on the interlayer interfacial normal stiffness for different angles of incidence and stacking sequences when $\varphi=90^{\circ}$ and $K_{\mathrm{T}}=\infty$. $\left(10^{\circ}<\theta<60^{\circ}\right)$ ranges mentioned above in Sec. III A, respectively. Likewise, the variation with $K_{\mathrm{T}}$ is depicted in Fig. 4 when $K_{\mathrm{N}}=\infty$ to look into the influence of interfacial shear stiffness. The vertical axes $K_{\mathrm{N}}{ }^{*} \equiv K_{\mathrm{N}} h / \operatorname{Re}\left[C_{33}\right]$ in Fig. 3 and $K_{\mathrm{T}}{ }^{*} \equiv K_{\mathrm{T}} h / \operatorname{Re}\left[C_{66}\right]$ in Fig. 4 represent the interfacial stiffnesses normalized by the ply thickness and stiffness, whose ranges correspond approximately to $0.01 \leq K_{\mathrm{N}} \leq 11 \mathrm{GPa} / \mu \mathrm{m}$ and $0.005 \leq K_{\mathrm{T}} \leq 5 \mathrm{GPa} / \mu \mathrm{m}$, respectively.

The transmission characteristics of the cross-ply laminate are shown in Figs. 3(a) and 4(a) for the incident angle $\theta=5^{\circ}$, and in Figs. 3(c) and 4(c) for $\theta=40^{\circ}$. In Fig. 3(a) when $K_{\mathrm{T}}=\infty$, the transmission coefficient drops to a low level at around $f^{*}=0.48$ when $K_{\mathrm{N}} *=10$ and its bandwidth becomes much wider as the normal stiffness decreases. In the case of finite shear stiffness in Fig. 4(a), totally different types of low-transmission zones from the one in Fig. 3(a) are generated such as those containing the points indicated by "C" and "D." The dependence of their bandwidth on the shear stiffness is, however, not as remarkable as that on the normal stiffness seen in Fig. 3(a).

When $\theta=40^{\circ}$ in Figs. 3(c) and 4(c), the normal and shear stiffnesses have a similar influence on the transmission coefficient when they are relatively large. For example, the low-transmission frequency ranges formed at around $f^{*}=0.3$ in Figs. 3(c) and 4(c) become narrower in a similar way when $K_{\mathrm{N}} *$ or $K_{\mathrm{T}} *$ decreases from $10^{2}$ to $10^{\circ}$. In contrast, when the interfacial stiffnesses fall to a lower level, say, $K_{\mathrm{N}} *<10^{\circ}$ and $K_{\mathrm{T}}^{*}<10^{\circ}$, the transmission characteristics are dependent separately on the normal and shear stiffnesses. In particular, the normal stiffness has a distinct effect to produce new low-transmission zones such as the one containing the point "J" in Fig. 3(c).

The corresponding results for the quasi-isotropic laminate are shown in Figs. 3(b) and 4(b) for the incident angle $\theta=5^{\circ}$, and in Figs. 3(d) and 4(d) for $\theta=40^{\circ}$. The influence
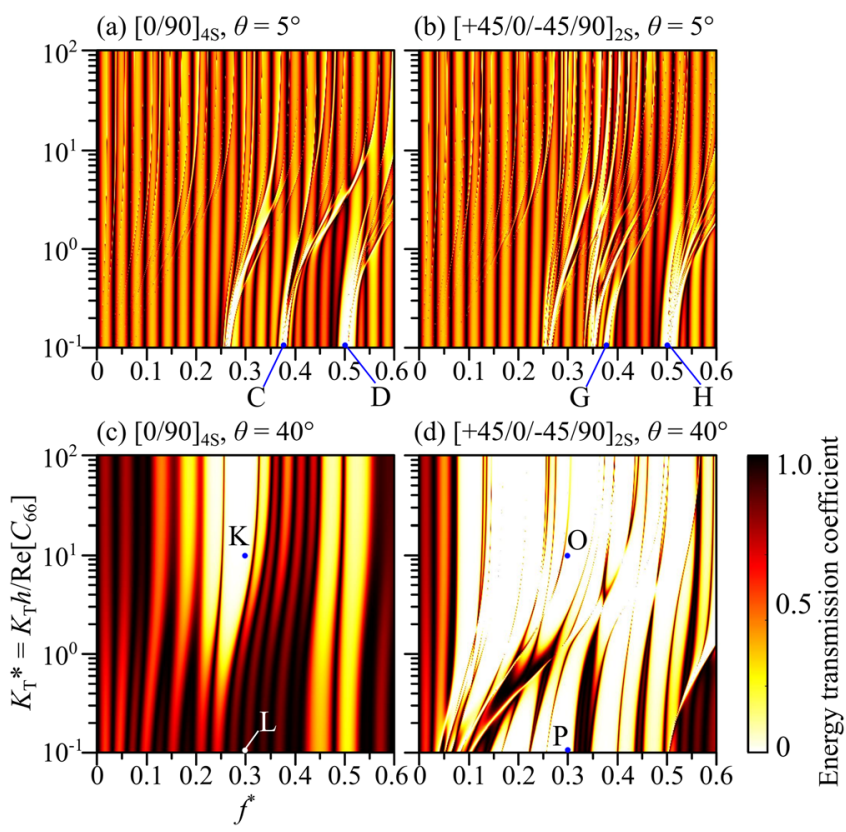

FIG. 4. (Color online) Dependence of the energy transmission spectrum on the interlayer interfacial shear stiffness for different angles of incidence and stacking sequences when $\varphi=90^{\circ}$ and $K_{\mathrm{N}}=\infty$. 
of stacking sequence on the stiffness dependence of wave transmissivity is not so significant for the smaller angle of incidence $\theta=5^{\circ}$ when Figs. 3(a) and 3(b) [Figs. 4(a) and 4(b)] are compared. On the other hand, it varies with the interfacial stiffnesses in a manner depending on the laminate lay-up for the larger angle of incidence $\theta=40^{\circ}$ when Figs. 3(c) and 3(d) [Figs. 4(c) and 4(d)] are compared. Unlike the cross-ply laminate in Fig. 4(c), other low-transmission zones are newly formed for the quasi-isotropic laminate even in the range of lower shear stiffness, such as the one containing the point " $P$ " in Fig. 4(d).

\section{DISCUSSIONS}

In Sec. III, the energy transmission coefficient has been shown to drop to a low level in certain finite frequency ranges depending on the angle of incidence, the stacking sequence, and the interlayer interfacial normal and shear stiffnesses. In order to investigate the formation mechanism of these lowtransmission zones seen in Figs. 2-4 in more detail, the complex Poynting vector ${ }^{52}$ of partial waves inside the laminate is examined here. The real part of the $x_{3}$ component of this vector, denoted by $P_{3}$, represents the time average of the energy flux density in the layering direction. In this section, this quantity is calculated for each partial wave inside the laminate according to the procedure outlined in Appendix A. Note that the previous studies ${ }^{1,53}$ analyzed the wave propagation behavior in infinitely periodic structures in terms of the energy flux density of the so-called Floquet waves. In contrast, the following analysis focuses on the energy flux density of partial waves as the present interest is to examine the wave propagation behavior in finite laminate structures.

\section{A. Distribution of energy flux density in the stacking direction}

\section{Small angle of incidence}

The distribution of $\operatorname{Re}\left[P_{3}\right]$ along the $x_{3}$ direction is depicted in Fig. 5 for the eight selected points corresponding to the small angle of incidence $\theta=5^{\circ}$, which are indicated by " $A$ " in Fig. 2(a), "B" in Fig. 3(a), "C" and "D" in Fig. 4(a), "E" in Fig. 2(b), "F" in Fig. 3(b), and "G" and " $H$ " in Fig. 4(b) as summarized in Table II. The wave transmission is partially achieved at the points " $A$ " and "E," while it is strongly prohibited at the others.

In Fig. 5, "QL+," "QT1+," and "QT2+" ("QL-," "QT1-," and "QT2-") denote the energy fluxes of the quasilongitudinal, fast quasi-transverse, and slow quasi-transverse waves propagating in the positive (negative) $x_{3}$ direction, respectively. Note that "QL+" and "QL-" include the pure longitudinal mode in water. The horizontal axis in Fig. 5 represents the energy flux density normalized by that of the incident wave given as $\left(\rho_{\mathrm{f}} V_{\mathrm{f}} \omega^{2} A_{\text {inc }}^{2} \cos \theta\right) / 2$. The vertical axis represents the $x_{3}$ coordinate normalized by the ply thickness, where $\left(x_{3}-Z_{0}\right) / h=0$ and $\left(x_{3}-Z_{0}\right) / h=-16$ correspond to the top and bottom surfaces of the laminate, respectively.

In Fig. 5, the energy flux densities remain constant in each ply since the viscoelastic nature of plies is neglected.
$[0 / 90]_{4 \mathrm{~S}}$

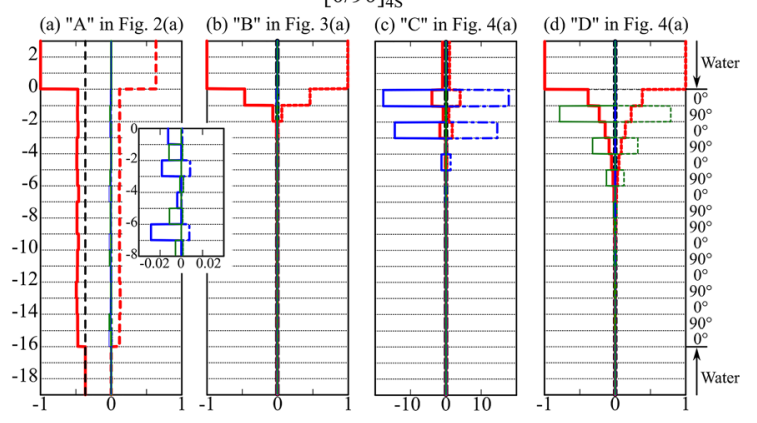

$[+45 / 0 /-45 / 90]_{2 S}$

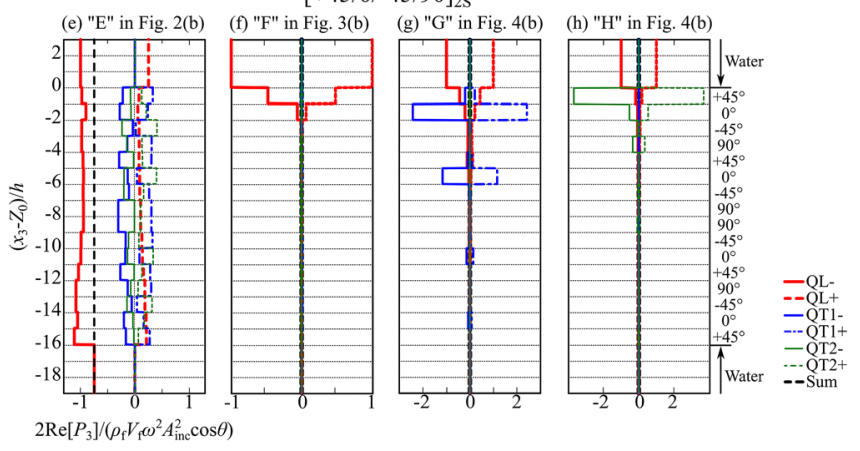

FIG. 5. (Color online) Distribution of energy flux density in the stacking direction for the points indicated by "A"-"H" in Figs. 2(a), 2(b), 3(a), 3(b), 4(a), and 4(b).

Furthermore, the conservation of energy can be confirmed, as the sum of the energy flux densities of all partial waves remains constant along the $x_{3}$ direction. In Fig. 5(a), the QL mode is dominant inside the cross-ply laminate with the perfectly bonded interlayer interfaces. Since the plane of incidence coincides with the plane of symmetry of the $0^{\circ}$ and $90^{\circ}$ plies, the out-of-plane modes (QT2 for the $0^{\circ}$ ply and QT1 for the $90^{\circ}$ ply) are not generated in the cross-ply laminate as shown in the inset of Fig. 5(a). In contrast, in the

TABLE II. Selected points in Figs. 2-4 for which the energy flux density distribution is calculated.

\begin{tabular}{|c|c|c|c|c|c|}
\hline \multirow[b]{2}{*}{ Symbol } & \multirow[b]{2}{*}{ Lay-up } & \multirow{2}{*}{$\begin{array}{c}\text { Angle of } \\
\text { incidence } \\
\theta\left[^{\circ}\right]\end{array}$} & \multirow{2}{*}{$\begin{array}{l}\text { Normalized } \\
\text { frequency } f^{*}\end{array}$} & \multicolumn{2}{|c|}{$\begin{array}{l}\text { Normalized interlayer } \\
\text { interfacial stiffnesses }\end{array}$} \\
\hline & & & & $K_{\mathrm{N}}^{*}$ & $K_{\mathrm{T}}^{*}$ \\
\hline A & {$[0 / 90]_{4 \mathrm{~S}}$} & 5 & 0.5 & $\infty$ & $\infty$ \\
\hline B & {$[0 / 90]_{4 \mathrm{~S}}$} & 5 & 0.4 & 1 & $\infty$ \\
\hline $\mathrm{C}$ & {$[0 / 90]_{4 \mathrm{~S}}$} & 5 & 0.38 & $\infty$ & 0.1 \\
\hline $\mathrm{D}$ & {$[0 / 90]_{4 \mathrm{~S}}$} & 5 & 0.5 & $\infty$ & 0.1 \\
\hline $\mathrm{E}$ & {$[+45 / 0 /-45 / 90]_{2 \mathrm{~S}}$} & 5 & 0.5 & $\infty$ & $\infty$ \\
\hline $\mathrm{F}$ & {$[+45 / 0 /-45 / 90]_{2 \mathrm{~S}}$} & 5 & 0.4 & 1 & $\infty$ \\
\hline G & {$[+45 / 0 /-45 / 90]_{2 \mathrm{~S}}$} & 5 & 0.38 & $\infty$ & 0.1 \\
\hline $\mathrm{H}$ & {$[+45 / 0 /-45 / 90]_{2 \mathrm{~S}}$} & 5 & 0.5 & $\infty$ & 0.1 \\
\hline I & {$[0 / 90]_{4 \mathrm{~S}}$} & 40 & 0.3 & 20 & $\infty$ \\
\hline $\mathrm{J}$ & {$[0 / 90]_{4 \mathrm{~S}}$} & 40 & 0.3 & 0.2 & $\infty$ \\
\hline $\mathrm{K}$ & {$[0 / 90]_{4 \mathrm{~S}}$} & 40 & 0.3 & $\infty$ & 10 \\
\hline $\mathrm{L}$ & {$[0 / 90]_{4 \mathrm{~S}}$} & 40 & 0.3 & $\infty$ & 0.1 \\
\hline M & {$[+45 / 0 /-45 / 90]_{2 \mathrm{~S}}$} & 40 & 0.3 & 20 & $\infty$ \\
\hline $\mathrm{N}$ & {$[+45 / 0 /-45 / 90]_{2 \mathrm{~S}}$} & 40 & 0.3 & 0.2 & $\infty$ \\
\hline $\mathrm{O}$ & {$[+45 / 0 /-45 / 90]_{2 \mathrm{~S}}$} & 40 & 0.3 & $\infty$ & 10 \\
\hline $\mathrm{P}$ & {$[+45 / 0 /-45 / 90]_{2 \mathrm{~S}}$} & 40 & 0.3 & $\infty$ & 0.1 \\
\hline
\end{tabular}


quasi-isotropic laminate in Fig. 5(e), not only the in-plane but also the out-of-plane mode is generated and the QT1 and QT2 modes account for a larger portion of energy compared to the cross-ply laminate in Fig. 5(a). Nevertheless, the energy distribution inside the laminate in Fig. 5(e) is still dominated by the QL mode. This dominant mode propagates and is polarized in almost the same direction regardless of the fiber direction of plies when $\theta=5^{\circ}$, as the deviation of wave vector (polarization direction) from the $x_{3}$ axis is $10.8^{\circ}$ $\left(19.5^{\circ}\right), 10.8^{\circ}\left(13.9^{\circ}\right)$, and $10.7^{\circ}\left(10.7^{\circ}\right)$ in the $0^{\circ}, \pm 45^{\circ}$, and $90^{\circ}$ plies, respectively. The wave transmissivity for small angles of incidence is, hence, not influenced by the stacking sequence very much as mentioned in Sec. III A regarding the results shown in Fig. 2.

When the interlayer interfaces possess a finite normal stiffness, the energy flux density distributions are shown in Figs. 5(b) and 5(f), where the energy transmission is very low for both cross-ply and quasi-isotropic laminates. In these cases, however, a few plies close to the upper surface contain similar non-zero energy fluxes of the QL mode. This indicates that the wave interference takes place for both laminates in a similar manner by the upward- and downward-propagating QL modes scattered from the interlayer interfaces. Namely, the corresponding low-transmission zones are the bandgaps caused by the QL mode. It is noted that the energy flux density of partial waves will vanish throughout the laminate if the low transmissivity is due to the total reflection at the waterlaminate interface, as the Poynting vector component becomes pure imaginary for evanescent waves.

When the interfacial shear stiffness has a finite value in Figs. 5(c) and 5(g) where both laminates lie in different low-transmission zones from Figs. 5(b) and 5(f), the energy distribution along the $x_{3}$ direction is dependent on the stacking sequence. There are still common features between Figs. 5(c) and 5(g) that the corresponding low-transmission zones are the bandgaps caused by the interference of QL and QT1 modes in combination, and that the QT1 mode becomes particularly remarkable in the $0^{\circ}$ plies. When the frequency increases from the point "C" [Fig. 5(c)] to "D" [Fig. 5(d)], or from the point "G" [Fig. 5(g)] to "H" [Fig. 5(h)], it still lies in different low-transmission zones, and the QT2 mode undertakes a role in the bandgap formation instead of the QT1 mode.

\section{Large angle of incidence}

For the angle of incidence $\theta=40^{\circ}$, the distribution of energy flux densities for different interfacial stiffnesses with a fixed frequency $f^{*}=0.3$ is shown in Fig. 6 for the eight selected points indicated by "I" and "J" in Fig. 3(c), "K" and "L" in Fig. 4(c), "M" and "N" in Fig. 3(d), and "O" and "P" in Fig. 4(d) as summarized in Table II. All these points lie in the low-transmission zones except for the point "L." For the same reason mentioned in Sec. IV A 1, these zones are found to be the bandgaps due to the wave interference.

For this angle of incidence, the energy flux of the QL mode vanishes throughout the laminate since it becomes evanescent in all the plies. As a result, the wave interference is caused by the quasi-transverse modes. In particular, the
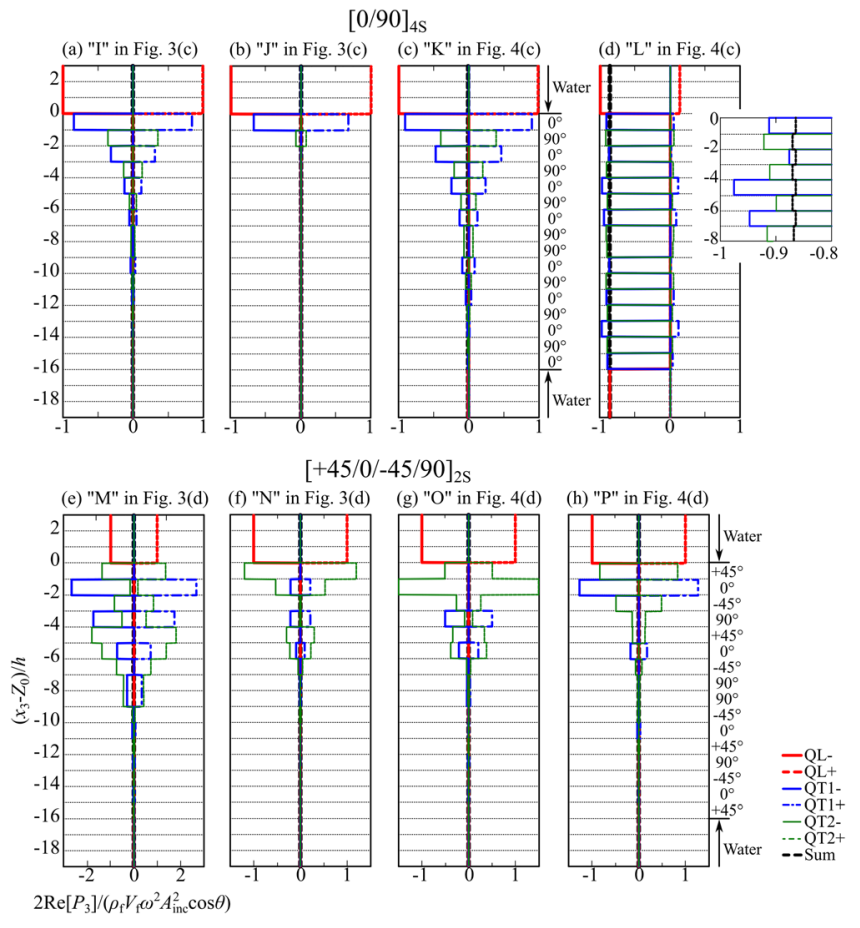

FIG. 6. (Color online) The same as Fig. 5, but for the points indicated by "I"-"P" in Figs. 3(c), 3(d), 4(c), and 4(d).

bandgaps of cross-ply laminate in Figs. 6(a)-6(c) are solely governed by the QT1 and QT2 modes in the $0^{\circ}$ and $90^{\circ}$ plies, respectively. In the case of the quasi-isotropic laminates in Figs. 6(e)-6(h), both of the QT1 and QT2 modes exist in the $0^{\circ}$ and $90^{\circ}$ plies, while only the QT2 mode has a non-zero flux in the $\pm 45^{\circ}$ plies since the QT1 mode is evanescent therein.

It should be noted that the sum of energy flux densities of the partial waves is no longer constant along the $x_{3}$ direction as seen in the inset of Fig. 6(d). This is because of the evanescent nature of the partial waves, which does not appear in the case of small angle of incidence in Sec. IV A 1. If the coupling effect ${ }^{52}$ of evanescent waves localized at the upper and lower edges of the ply is incorporated by considering the energy flux for a pair of upward- and downwardpropagating partial waves (Appendix B), the resulting fluxes vanish almost completely throughout the thickness direction for the low-transmission zones even when the wave interference occurs.

Consequently, for the two angles of incidence examined here, it can be concluded that the finite frequency ranges of low transmissivity presented in Sec. III are the bandgaps due to the wave interference inside the laminate. The mode combination causing such interference is influenced profoundly by the angle of incidence, the stacking sequence, and the interlayer interfacial normal and shear stiffnesses.

\section{B. Comparison with Floquet wave}

For unidirectional composite laminates with spring-type interlayer interfaces, the previous $\operatorname{study}^{48}$ has shown that the wave transmission characteristics of finite laminate structure are closely related to the propagation behavior of the Floquet waves in the infinitely extended laminate structure. Following this result, the correspondence between finite and 
infinite laminate structures is examined here for the wave propagation behavior in the cross-ply and quasi-isotropic lay-ups by comparing the energy flux density distributions inside the structures and calculating the number of propagative Floquet modes.

In infinitely extended periodic multilayered structures, the wave propagation is characterized by three pairs of Floquet wave modes propagating in the opposite $x_{3}$ directions. ${ }^{48}$ Each Floquet mode is a superposition of classical plane partial waves in each layer. ${ }^{27} \mathrm{By}$ assuming that the $x_{1}$ and $x_{2}$ components are $-k_{\mathrm{f}} \sin \theta$ and zero, respectively, the $x_{3}$ components of the wavenumber of the Floquet waves can be calculated according to Appendix C. The distribution of the energy flux densities of the plane partial waves constituting the Floquet waves propagating (or decaying) in the negative $x_{3}$ direction is shown in Fig. 7 for the two selected points indicated by "B" in Fig. 3(a) and "P" in Fig. 4(d): the corresponding results for the finite laminate structures are shown in Figs. 5(b) and 6(h), respectively. Note that Fig. 7 exhibits the energy flux when a single Floquet mode is decomposed into the six partial waves in each layer, not the energy flux of Floquet wave itself as considered in Refs. 1 and 53. Figures 7(a)-7(c) and Figs. 7(d)-7(f) represent three Floquet modes for the infinitely periodic structures whose unit-cells are $[0 / 90]$ and $[+45 / 0 /-45 / 90]$, respectively. For both layups, the energy distribution is depicted for eight plies, i.e., four unit-cells in Figs. 7(a)-7(c) and two unit-cells in Figs. 7(d)-7(f). The energy flux densities in Fig. 7 are normalized by the sum for six partial waves when the Floquet mode is propagative, i.e., the Floquet wavenumber is real [Figs. 7(b) and $7(\mathrm{c})$ ] and by the maximum value when the Floquet mode is non-propagative, i.e., the Floquet wavenumber possesses a negative imaginary part [Figs. 7(a) and 7(d)-7(f)], respectively.

The cross-ply laminate at the point " $\mathrm{B}$ " is characterized by one non-propagative and two propagative Floquet modes. The first non-propagative Floquet mode in Fig. 7(a) is found to have a similar energy distribution pattern to that of the immersed finite cross-ply laminate in Fig. 5(b). The other Floquet modes are dominated either by the out-of-plane modes [Fig. 7(b)] or by the quasi-transverse modes [Fig. 7(c)]. These modes do not have their counterparts in Fig. 5(b) as
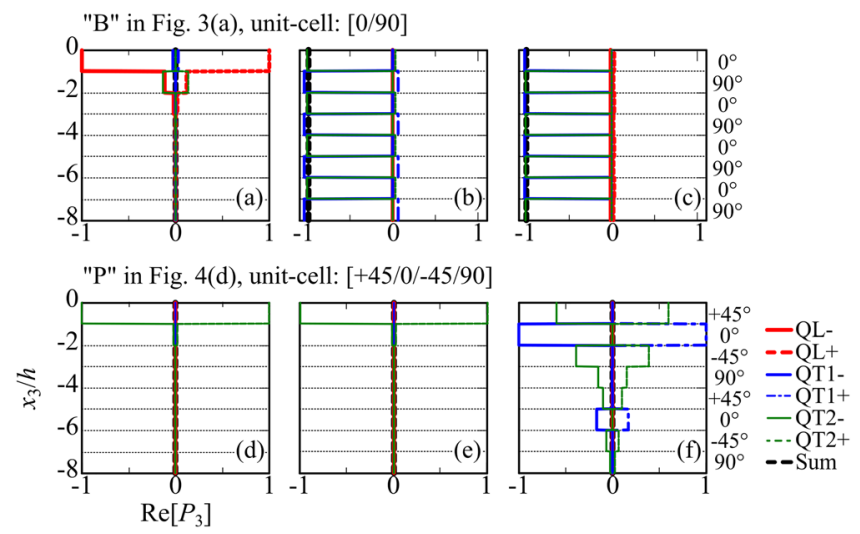

FIG. 7. (Color online) Distribution of the normalized energy flux density of three downward-propagating Floquet waves for the points indicated by (a)-(c) "B" in Fig. 3(a) and (d)-(f) "P" in Fig. 4(d). they do not couple significantly with the longitudinal wave impinging on the immersed finite laminate structure at this small angle of incidence $\theta=5^{\circ}$. The quasi-isotropic laminate at the point "P" is characterized by three non-propagative Floquet modes. The two Floquet modes in Figs. 7(d) and 7(e) have very similar energy distribution dominated by the QT2 mode, which is attenuated almost completely in the first $+45^{\circ}$ ply, while the distribution pattern in Fig. 7(f) can be favorably compared with that of the finite quasi-isotropic laminate in Fig. 6(h).

With the same parameters used for the computation of the transmission coefficients in Figs. 3(a) and 4(d), the number of pairs of propagative Floquet modes of corresponding infinitely laminate structures is counted as in Ref. 48 and illustrated in Figs. 8(a) and 8(b), respectively. When comparing Figs. 3(a) and 8(a) for the finite and infinite cross-ply laminates, the patterns of low-transmission zones such as those containing the point " $\mathrm{B}$ " in Fig. 3(a) are favorably observable in Fig. 8(a) where the Floquet mode possessing the energy flux distribution similar to that of the finite laminate becomes non-propagative. Note that as shown in Fig. 7 , the wave field inside the immersed finite laminate is mainly governed by a single Floquet mode, so the transmission coefficient is not sensitive to whether the other two Floquet modes are propagative or not. In Fig. 8(b) for the infinite quasi-isotropic laminate corresponding to the large angle of incidence $\theta=40^{\circ}$, the number of propagative Floquet waves becomes zero or one over a wide area, and white zones are fairly compared with the low-transmission zones of finite laminate in Fig. 4(d).

It can be thus reasonably expected that the ultrasonic wave causes the interference even in the immersed finite laminate structure in almost the same manner as one out of three Floquet modes does in the corresponding infinite laminate structure. Note that since the energy fluxes vanish almost entirely in the upper half of the laminate when the interference occurs in Figs. 5 and 6, the influence of symmetric stacking of layers is insignificant in the present comparison between finite and infinite laminate structures.

\section{COMPARISON WITH MEASUREMENT}

The measurement of the energy transmission spectrum was carried out for 16-ply cross-ply $[0 / 90]_{4 \mathrm{~S}}$ and quasi-isotropic $[+45 / 0 /-45 / 90]_{2 s}$ composite laminates
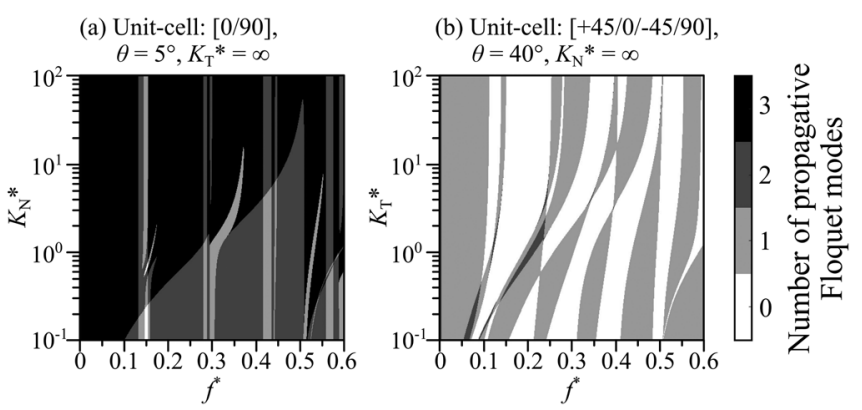

FIG. 8. The number of propagative Floquet modes of infinitely laminated structures (a) in the $f^{*}-K_{\mathrm{N}} *$ plane when the unit-cell is [0/90] and (b) in the $f^{*}-K_{\mathrm{T}} *$ plane when the unit-cell is $[+45 / 0 /-45 / 90]$. 
(thickness: $2.16 \mathrm{~mm}$ ) immersed in water. The specimens were made of carbon/epoxy composite plies (carbon fibers TR30 and epoxy resin \#340, Mitsubishi Rayon, Co. Ltd., Tokyo, Japan) and had the same ply thickness of about $0.135 \mathrm{~mm}$. The experimental procedure follows Ref. 48, except that the through-transmission measurement was performed instead of the double-through transmission measurement in Ref. 48. A piezoelectric broadband transducer of nominal frequency $10 \mathrm{MHz}$ with a diameter 0.5 in. (IS1013R, Insight, Inc., Tokyo, Japan) was used as the transmitter. The ultrasonic wave transmitted through a specimen was received at the other side by another piezoelectric broadband transducer of the same nominal frequency but with a larger diameter 1 in. (IY1025R, Insight, Inc., Tokyo, Japan). Compared to the double through-transmission technique used in Ref. 48, the through-transmission technique is advantageous to reduce the effect of wave attenuation. With this method, care should be taken of the fact that the beam sound axis of the first arrival transmitted waves, as well as the following ones due to the multiple reflections inside the specimen, is shifted from that of the incident wave due to the refraction effect. The influence of such finite beam size can be accounted for, for example, by shifting the position of the receiver in the horizontal direction ${ }^{54}$ to obtain the results to be compared with the predictions of planewave theory. In the present measurement, however, the position of the receiver was fixed on the beam sound axis of the incident wave since the laminates were so thin that the beam axis shifts were minimal. In addition, the receiving transducer with larger diameter is also a solution to remove as much influence of beam refraction as possible. Furthermore, the two transducers were separated by about $50 \mathrm{~mm}$, which was much larger than the thickness of specimens, so the influence of beam diffraction can be reasonably suppressed as the spectrum of transmitted signals was normalized by that of the reference wave measured without the specimen to obtain the transmission coefficient.

The variation of the measured energy transmission spectrum with the angle of incidence is shown in Figs. 9(a)-9(f) for $\varphi=0^{\circ}, 45^{\circ}$, and $90^{\circ}$, where $\varphi=0^{\circ}$ corresponds to the plane normal to the fiber direction of the $0^{\circ}$ plies. Note that a logarithmic scale is used for the color bar to make the spectrum in larger angles of incidence of $\theta>10^{\circ}$ conspicuous. Because of the limited bandwidth of the transducers, the results are shown in Fig. 9 for a finite frequency range of $3 \leq f \leq 13 \mathrm{MHz}$. The theoretical results are also depicted in Figs. 9(g)-9(1) for comparison. These are calculated by the stiffness-matrix method using the properties in Table I, including the imaginary parts of the ply stiffness with the interlayer interfacial stiffnesses $K_{\mathrm{N}}=3.0 \mathrm{GPa} / \mu \mathrm{m}$ and $K_{\mathrm{T}}=0.8 \mathrm{GPa} / \mu \mathrm{m}$. These laminate as well as interface parameters were determined from the transmission coefficient data measured in Ref. 48 for a unidirectional composite laminate made of the same materials as the present specimens. It is noted that the ply stiffness

\section{Experiment}

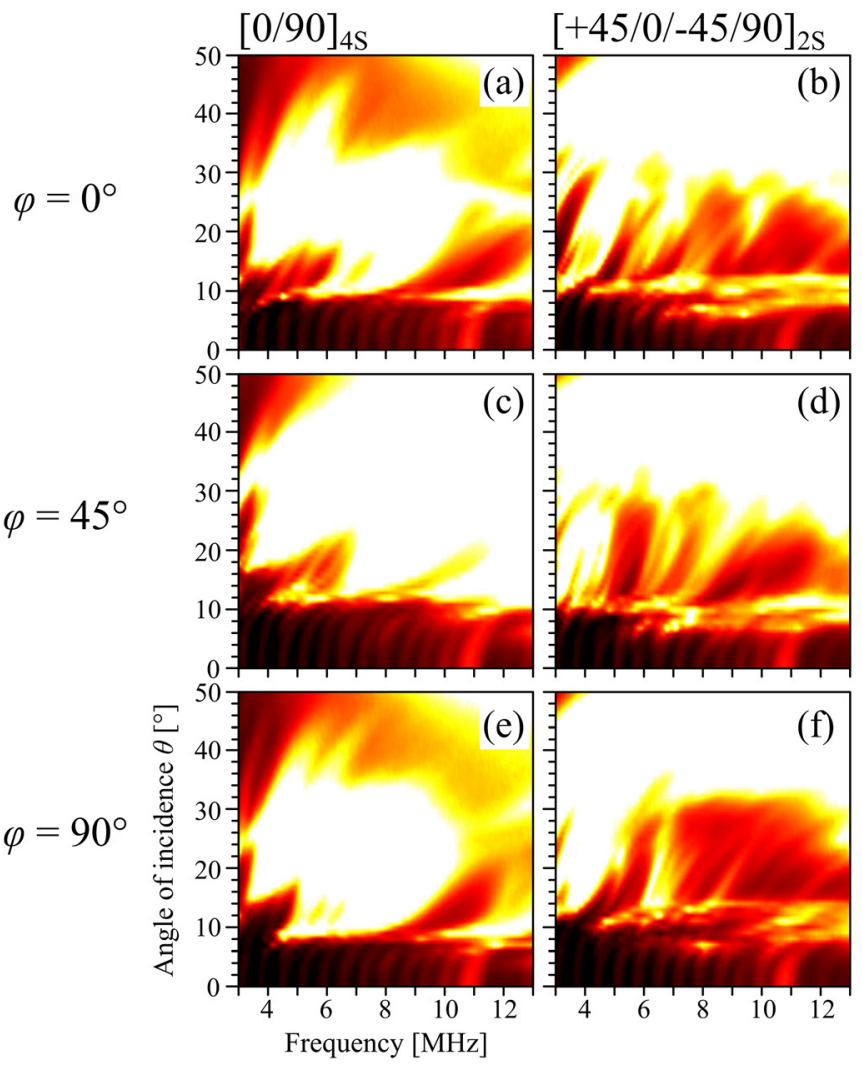

Theory
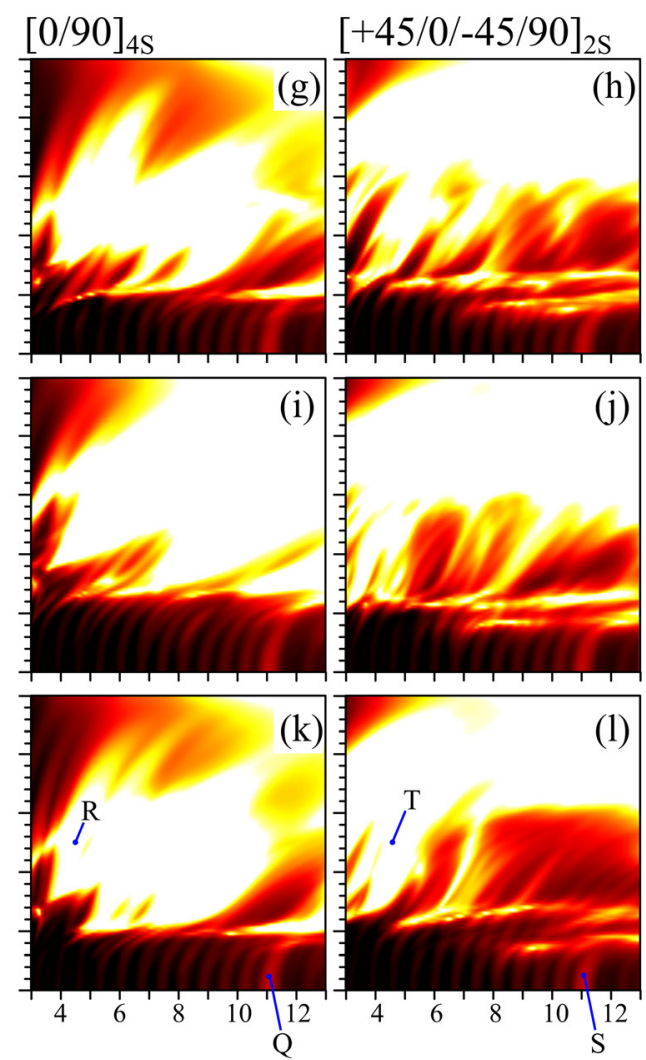

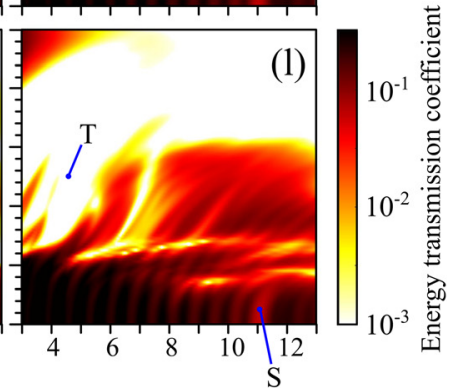

FIG. 9. (Color online) Variation of (a)-(f) experimental and (g)-(1) theoretical energy transmission spectrum of 16-ply carbon/epoxy cross-ply and quasiisotropic composite laminates with the angle of incidence when $\varphi=0^{\circ}, 45^{\circ}$, and $90^{\circ}$. 
values shown in Table I are slightly different from those in Ref. 48: in Ref. 48, these parameters were determined so that the theoretical transmission coefficients in the $f-\theta$ plane fit best to the experimental ones for $\varphi=0^{\circ}, 45^{\circ}$, and $90^{\circ}$, while in the present study the same evaluation procedure was performed using the larger set of data for $0^{\circ} \leq \varphi \leq 90^{\circ}$ with the increment $\Delta \varphi=3^{\circ}$. As mentioned in Sec. III B, the interfacial stiffnesses and the material properties of resin-rich regions are simply related by $K_{\mathrm{N}}=\left(\lambda_{\mathrm{e}}+2 \mu_{\mathrm{e}}\right) / h_{\mathrm{e}}$ and $K_{\mathrm{T}}=\mu_{\mathrm{e}} / h_{\mathrm{e}}$. Based on this relation with the elastic constants measured for the bulk epoxy resin $\# 340, \lambda_{\mathrm{e}}+2 \mu_{\mathrm{e}}=9.3 \mathrm{GPa}$ and $\mu_{\mathrm{e}}=1.8 \mathrm{GPa},{ }^{51}$ the interfacial stiffnesses identified above imply an equivalent thickness of resin-rich regions $2.3-3.1 \mu \mathrm{m}$, which is considered to be reasonable in comparison with the one measured for the present cross-ply laminate by the micrographic observation $3-8 \mu \mathrm{m},{ }^{46}$ as the resin-rich regions of unidirectional laminates are likely to be thinner than those of multidirectional laminates.

For approximately $\theta<10^{\circ}$ in Figs. 9(a) -9 (f), the bandgaps are seen for both laminates at around $11 \mathrm{MHz}$ regardless of the angle $\varphi$, which conforms to the result in Sec. IV A 1 that the wave transmissivity is not influenced by the stacking sequence very much for small angles of incidence. For large angles of incidence, i.e., $10^{\circ}<\theta<40^{\circ}$ for the cross-ply laminate and $10^{\circ}<\theta<30^{\circ}$ for the quasi-isotropic laminate, the bandgaps can be observed, e.g., at around $4 \mathrm{MHz}$ and $6.5 \mathrm{MHz}$ for $\theta=20^{\circ}$ in Fig. 9(f), although the boundaries between neighboring bandgaps are not as clear as in the theoretical results in Fig. 2 due mainly to the ply viscoelastic nature. The transmission characteristics and the bandgap formation seen in the $f-\theta$ plane and their dependence on the angle $\varphi$ in the experimental results are favorably reproduced by the theory in Figs. 9(g)-9(l). The significance of incorporating the influence of imperfect interlayer interfaces can be verified by comparing Figs. 2 and 9, as the bandgaps for $\theta<10^{\circ}$ observed at $11 \mathrm{MHz}$ in the experimental results are not reproduced in the case of perfectly bonded interfaces in Fig. 2.

Using the above-mentioned material properties, the distribution of energy flux density inside the cross-ply and quasiisotropic laminates immersed in water is calculated for the points indicated by "Q"-"T" in Figs. 9(k) and 9(l), and depicted in Fig. 10. These points exhibit relatively low energy transmissivities and correspond to the bandgaps. It should be noted that the energy fluxes of partial waves in Fig. 10 show decreasing behavior in each ply due to the ply viscoelastic nature, in contrast to Figs. 5 and 6 where they are constant.

Figures 10(a) and 10(c) show the energy distributions when $\theta=3^{\circ}$ and $f=11 \mathrm{MHz}$. The corresponding bandgaps are found to be due to the wave interference of the QL mode for both cross-ply and quasi-isotropic laminates. The decay of the QL mode in the layering direction is relatively weak in these two cases, as the wave scattering at the interlayer interfaces are not so significant due to the high interfacial stiffnesses $K_{\mathrm{N}}{ }^{*}=27$ and $K_{\mathrm{T}}{ }^{*}=17$. In Figs. 10(b) and 10(d) for $\theta=25^{\circ}$ and $f=4.5 \mathrm{MHz}$, the wave interference is caused by the quasi-transverse modes dominantly since the QL mode is propagative only in the $90^{\circ}$ plies. In particular, the bandgap of cross-ply laminate in Fig. 10(b) is governed by
$[0 / 90]_{4 S}$
(a) "Q" in Fig. 9(k)

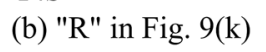

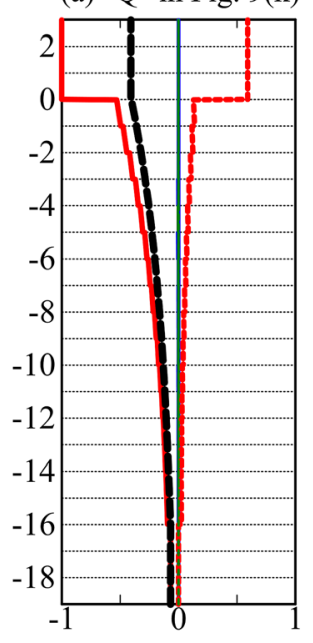

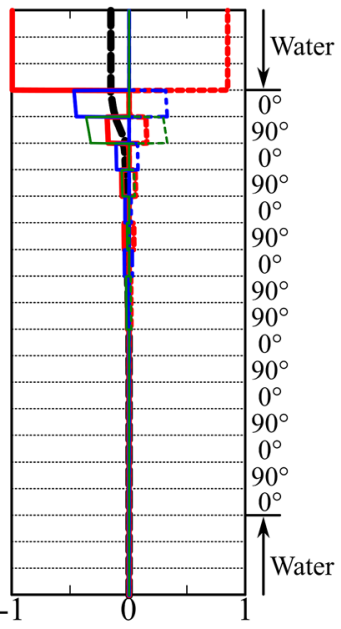

$$
[+45 / 0 /-45 / 90]_{2 \mathrm{~S}}
$$

(c) "S" in Fig. 9(1)

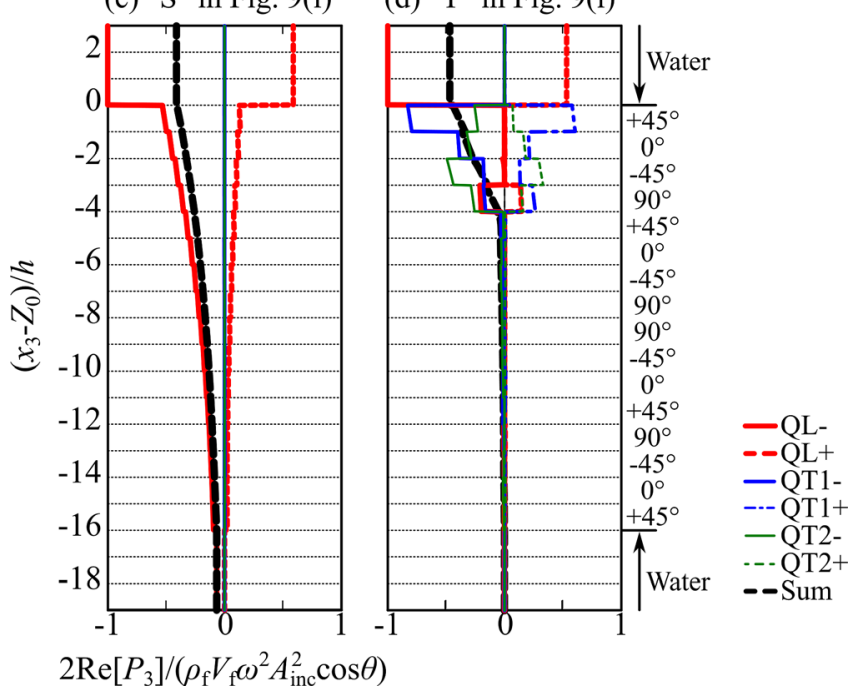

FIG. 10. (Color online) Distribution of energy flux density in the stacking direction for the points indicated by "Q"-"T" in Figs. 9(k) and 9(1). Note that the ply viscoelastic nature is considered.

the combination of QT1 mode in the $0^{\circ}$ plies and the QL and QT2 modes in the $90^{\circ}$ plies.

\section{SUMMARY}

In the present study, the ultrasonic wave transmission through cross-ply and quasi-isotropic composite laminates with spring-type interlayer interfaces has been analyzed theoretically by using the stiffness-matrix approach. It has been shown that the frequency ranges of finite bandwidth in which the energy transmission coefficient drops to vanishingly low levels are profoundly influenced by the angle of incidence, the stacking sequence of laminates, and the interlayer interfacial normal and shear stiffnesses. By calculating the energy flux density inside the laminate, these low-transmission zones have been shown to be the bandgaps due to the constructive interference of scattered waves from the interlayer interfaces. In addition, the mode combination causing the interference has been shown to vary 
remarkably with the frequency, the incident angle, the stacking sequence, and the interlayer interfacial stiffnesses. Furthermore, the wave interference in the finite laminate structure has been found to occur in almost the same manner as the Floquet wave does in the corresponding infinitely periodic structure. The energy transmission spectrum has been experimentally measured for 16-ply carbon/epoxy composite laminates with cross-ply and quasi-isotropic lay-ups for various incident directions. The observed transmission characteristics and the bandgap behavior have been favorably compared with the theory. The results of the present analysis can be helpful when the imperfect interlayer interfaces of multidirectional composite laminates are evaluated from the ultrasonic wave propagation characteristics.

\section{ACKNOWLEDGMENTS}

This work has been supported by JSPS (Japan Society for the Promotion of Science) KAKENHI Grant Nos. JP25-1754 and JP15K13833.

\section{APPENDIX A: CALCULATION OF POYNTING VECTOR COMPONENT INSIDE LAMINATE}

In what follows, the summation convention is not used to avoid confusion. The displacement and stress vectors at the top $\left(x_{3}=Z_{0,+}\right)$ and bottom $\left(x_{3}=Z_{N,-}\right)$ surfaces of the composite laminate immersed in water can be written as

$$
\begin{aligned}
& \left.\mathbf{u}\right|_{x_{3}=Z_{0,+}}=A_{\text {inc }}\left(\begin{array}{c}
(1+R) \sin \theta \\
0 \\
(1-R) \cos \theta
\end{array}\right), \\
& \left.\boldsymbol{\sigma}\right|_{x_{3}=Z_{0,+}}=A_{\text {inc }}\left(\begin{array}{c}
0 \\
-i \rho_{\mathrm{f}} V_{\mathrm{f}} \omega(1+R)
\end{array}\right), \\
& \left.\mathbf{u}\right|_{x_{3}=Z_{N,-}}=A_{\text {inc }}\left(\begin{array}{c}
T \sin \theta \\
0 \\
T \cos \theta
\end{array}\right) e^{i k_{\mathrm{f}} H \cos \theta} \\
& 0 \\
& \left.\boldsymbol{\sigma}\right|_{x_{3}=Z_{N,-}}=A_{\text {inc }}\left(\begin{array}{c}
0 \\
-i \rho_{\mathrm{f}} V_{\mathrm{f}} \omega T
\end{array}\right) e^{i k_{\mathrm{f}} H \cos \theta},
\end{aligned}
$$

where the common term depending on $x_{1}$ and the time, $\exp \left[-i\left(k_{\mathrm{f}} x_{1} \sin \theta+\omega t\right)\right]$, is omitted, which is conserved throughout the laminate because of Snell's law. In the above expressions, $R$ is the complex amplitude reflection coefficient calculated by the stiffness-matrix method ${ }^{49,50}$ as

$$
R=\frac{\left(\gamma+S_{33}^{\mathrm{G}}\right)\left(\gamma+S_{66}^{\mathrm{G}}\right)-S_{36}^{\mathrm{G}} S_{63}^{\mathrm{G}}}{\left(\gamma-S_{33}^{\mathrm{G}}\right)\left(\gamma+S_{66}^{\mathrm{G}}\right)+S_{36}^{\mathrm{G}} S_{63}^{\mathrm{G}}} .
$$

Using Eqs. (A1)-(A4) with the local stiffness matrices of plies as well as interlayer interfaces in Eqs. (4) and (5), the stress vectors at the upper and lower edges of the $m$ th ply $(m=1,2,3, \ldots, N)$ can be calculated (refer to Ref. 50 for the detailed procedure). The complex-valued amplitude of partial waves in the $m$ th ply is then obtained by using the stress vectors at the upper $\left(x_{3}=Z_{m-1,-}\right)$ and lower $\left(x_{3}=Z_{m,+}\right)$ edges of the corresponding ply as

$$
\begin{aligned}
\left(\begin{array}{l}
A^{1-} \\
A^{2-} \\
A^{3-} \\
A^{1+} \\
A^{2+} \\
A^{3+}
\end{array}\right)= & {\left[\begin{array}{cc}
\mathbf{D}^{-} & \mathbf{D}^{+} \mathbf{H}^{+}\left(Z_{m-1}-Z_{m}\right) \\
\mathbf{D}^{-} \mathbf{H}^{-}\left(Z_{m-1}-Z_{m}\right) & \mathbf{D}^{+}
\end{array}\right]^{-1} } \\
& \times\left(\begin{array}{c}
\left.\boldsymbol{\sigma}\right|_{x_{3}=Z_{m-1,-}} \\
\left.\boldsymbol{\sigma}\right|_{x_{3}=Z_{m,+}}
\end{array}\right)
\end{aligned}
$$

where $A^{\mathrm{s}+}$ and $A^{\mathrm{s}-}(s=1,2,3)$ represent the amplitude of partial waves (quasi-longitudinal and two quasi-transverse modes) propagating in the $m$ th ply in the positive and negative $x_{3}$ directions, respectively. In Eq. (A6), $\mathbf{D}^{ \pm}$and $\mathbf{H}^{ \pm}$are $3 \times 3$ matrices given by

$$
\begin{gathered}
\mathbf{D}^{ \pm}=i \sum_{q=1}^{3} \sum_{j=1}^{3}\left[\begin{array}{lll}
C_{13 j q} p_{j}^{1 \pm} k_{q}^{1 \pm} & C_{13 j q} p_{j}^{2 \pm} k_{q}^{2 \pm} & C_{13 j q} p_{j}^{3 \pm} k_{q}^{3 \pm} \\
C_{23 j q} p_{j}^{1 \pm} k_{q}^{1 \pm} & C_{23 j q} p_{j}^{2 \pm} k_{q}^{2 \pm} & C_{23 j q} p_{j}^{3 \pm} k_{q}^{3 \pm} \\
C_{33 j q} p_{j}^{1 \pm} k_{q}^{1 \pm} & C_{33 j q} p_{j}^{2 \pm} k_{q}^{2 \pm} & C_{33 j q} p_{j}^{3 \pm} k_{q}^{3 \pm}
\end{array}\right], \\
\mathbf{H}^{ \pm}(z)=\left[\begin{array}{ccc}
\exp \left( \pm i k_{3}^{1 \pm} z\right) & 0 & 0 \\
0 & \exp \left( \pm i k_{3}^{2 \pm} z\right) & 0 \\
0 & 0 & \exp \left( \pm i k_{3}^{3 \pm} z\right)
\end{array}\right]
\end{gathered}
$$

where $C_{r 3 j q}(r, j, q=1,2,3)$ are the elastic constants of the $m$ th ply, and $p_{j}^{s \pm}$ and $k_{q}^{s^{ \pm}}$are the polarization and wave vectors of the partial waves in the $m$ th ply, respectively. The polarization and the $x_{3}$ component of wave vector can be obtained by solving the Christoffel equation with the known parameters $k_{1}^{s^{ \pm}}=-k_{\mathrm{f}} \sin \theta$ and $k_{2}^{s^{ \pm}}=0 .^{50}$

The $x_{3}$ component of complex Poynting vector ${ }^{52}$ for the partial waves propagating in the $m$ th ply in the negative and positive $x_{3}$ directions is then given as

$$
\begin{aligned}
P_{3}^{s-}\left(x_{3}\right)= & -\frac{1}{2} \sum_{j=1}^{3} \overline{v_{j}^{s-}\left(x_{3}\right)} \sigma_{j 3}^{s-}\left(x_{3}\right) \\
= & -\frac{1}{2} i \omega\left|A^{s-}\right|^{2} W_{s s}\left(x_{3}\right), \\
P_{3}^{s+}\left(x_{3}\right)= & -\frac{1}{2} \sum_{j=1}^{3} \overline{v_{j}^{s+}\left(x_{3}\right)} \sigma_{j 3}^{s+}\left(x_{3}\right) \\
= & -\frac{1}{2} i \omega\left|A^{s+}\right|^{2} W_{(s+3)(s+3)}\left(x_{3}\right), \\
& s=1,2,3, \quad Z_{m}<x_{3}<Z_{m-1},
\end{aligned}
$$

where "-" and " $|\cdot|$ " denote the complex conjugate and absolute value, respectively. In the above expressions, $v_{j}^{s^{ \pm}}$and $\sigma_{j 3}^{s \pm}$ are the velocity and stress components of the partial waves, and $W_{s s}$ are the elements of a $6 \times 6$ matrix given by 


$$
\mathbf{W}\left(x_{3}\right)=\left[\begin{array}{cc}
\overline{\mathbf{H}^{-}\left(Z_{m-1}-x_{3}\right)}\left(\mathbf{P}^{-}\right)^{\dagger} \mathbf{D}^{-} \mathbf{H}^{-}\left(Z_{m-1}-x_{3}\right) & \overline{\mathbf{H}^{-}\left(Z_{m-1}-x_{3}\right)}\left(\mathbf{P}^{-}\right)^{\dagger} \mathbf{D}^{+} \mathbf{H}^{+}\left(x_{3}-Z_{m}\right) \\
\overline{\mathbf{H}^{+}\left(x_{3}-Z_{m}\right)}\left(\mathbf{P}^{+}\right)^{\dagger} \mathbf{D}^{-} \mathbf{H}^{-}\left(Z_{m-1}-x_{3}\right) & \overline{\mathbf{H}^{+}\left(x_{3}-Z_{m}\right)}\left(\mathbf{P}^{+}\right)^{\dagger} \mathbf{D}^{+} \mathbf{H}^{+}\left(x_{3}-Z_{m}\right)
\end{array}\right],
$$

where the superscript " $\dagger$ " denotes the Hermitian transpose and $\mathbf{P}^{ \pm}$are $3 \times 3$ matrices given by

$$
\mathbf{P}^{ \pm}=\left[\begin{array}{lll}
p_{1}^{1 \pm} & p_{1}^{2 \pm} & p_{1}^{3 \pm} \\
p_{2}^{1 \pm} & p_{2}^{2 \pm} & p_{2}^{3 \pm} \\
p_{3}^{1 \pm} & p_{3}^{2 \pm} & p_{3}^{3 \pm}
\end{array}\right] .
$$

The Poynting vector inside the composite laminate can be obtained by carrying out the above computation [Eqs. (A6)-(A12)] for all plies constituting the laminate.

\section{APPENDIX B: POYNTING VECTOR INCORPORATING COUPLING EFFECT}

Equations (A9) and (A10) yield the Poynting vector component for a single partial wave. In this framework, the energy flux density $\left(\operatorname{Re}\left[P_{3}^{s^{ \pm}}\right]\right)$vanishes for evanescent modes. On the other hand, the evanescent modes localized at the upper and lower edges of the ply can in combination transfer the energy in the thickness direction. ${ }^{52}$ Such a coupling effect can be incorporated by calculating the Poynting vector component for a pair of upward- and downward-propagating partial waves as

$$
\begin{aligned}
& P_{3}^{s}\left(x_{3}\right)=-\frac{1}{2} \sum_{j=1}^{3}\left[\overline{v_{j}^{s-}\left(x_{3}\right)}+\overline{v_{j}^{s+}\left(x_{3}\right)}\right] \\
& \times\left[\sigma_{j 3}^{s-}\left(x_{3}\right)+\sigma_{j 3}^{s+}\left(x_{3}\right)\right] \\
& =-\frac{1}{2} i \omega\left[\overline{A^{s-}} \quad \overline{A^{s+}}\right] \\
& \times\left[\begin{array}{cc}
W_{s s}\left(x_{3}\right) & W_{s(s+3)}\left(x_{3}\right) \\
W_{(s+3) s}\left(x_{3}\right) & W_{(s+3)(s+3)}\left(x_{3}\right)
\end{array}\right] \\
& \times\left[\begin{array}{c}
A^{s-} \\
A^{s+}
\end{array}\right], \quad s=1,2,3 .
\end{aligned}
$$

\section{APPENDIX C: POYNTING VECTOR IN INFINITELY PERIODIC STRUCTURE}

The $x_{3}$ component of Floquet wavenumber for an infinitely periodic structure, denoted by $\zeta$, can be calculated by solving ${ }^{48}$

$$
\operatorname{det}\left[\mathbf{K}_{21} \exp \left(i \zeta h_{\mathbf{u}}\right)-\mathbf{K}_{12} \exp \left(-i \zeta h_{\mathrm{u}}\right)+\mathbf{K}_{22}-\mathbf{K}_{11}\right]=0,
$$

where $h_{\mathrm{u}}$ is the thickness of a unit-cell of the periodic structure, and $\mathbf{K}_{I J}(I, J=1,2)$ are the $3 \times 3$ submatrices of the $6 \times 6$ stiffness matrix for the unit-cell. Note that Eq. (C1) has six solutions for $\zeta$ corresponding to three pairs of Floquet modes propagating in the opposite $x_{3}$ directions. ${ }^{48}$
For each Floquet mode, the displacement and stress vectors at the upper $\left(x_{3}=Z_{\mathrm{U}}\right)$ and lower $\left(x_{3}=Z_{\mathrm{L}}=Z_{\mathrm{U}}-h_{\mathrm{u}}\right)$ surfaces of the unit-cell can be calculated from the following equations with the so-obtained $\zeta$ from Eq. (C1):

$$
\begin{aligned}
& {\left[\mathbf{K}_{21} \exp \left(i \zeta h_{\mathrm{u}}\right)-\mathbf{K}_{12} \exp \left(-i \zeta h_{\mathrm{u}}\right)\right.} \\
& \left.\quad+\mathbf{K}_{22}-\mathbf{K}_{11}\right]\left.\mathbf{u}\right|_{x_{3}=Z_{\mathrm{L}}}=\mathbf{0}, \\
& \left.\mathbf{u}\right|_{x_{3}=Z_{\mathrm{U}}}=\left.\exp \left(i \zeta h_{\mathrm{u}}\right) \mathbf{u}\right|_{x_{3}=Z_{\mathrm{L}}}, \\
& \left(\begin{array}{l}
\left.\boldsymbol{\sigma}\right|_{x_{3}=Z_{\mathrm{U}}} \\
\left.\boldsymbol{\sigma}\right|_{x_{3}=Z_{\mathrm{L}}}
\end{array}\right)=\left[\begin{array}{ll}
\mathbf{K}_{11} & \mathbf{K}_{12} \\
\mathbf{K}_{21} & \mathbf{K}_{22}
\end{array}\right]\left(\begin{array}{l}
\left.\mathbf{u}\right|_{x_{3}=Z_{\mathrm{U}}} \\
\left.\mathbf{u}\right|_{x_{3}=Z_{\mathrm{L}}}
\end{array}\right) .
\end{aligned}
$$

Using the obtained displacement and stress vectors, the Poynting vector of partial waves constituting the corresponding Floquet wave can be calculated in the same manner as described in Appendix A.

It should be noted that solving Eqs. $(\mathrm{C} 1)-(\mathrm{C} 4)$ for $\zeta$ and the corresponding displacement and stress vectors is equivalent to calculating the eigenvalues and the corresponding eigenvectors of the transfer matrix of the unit-cell as in Ref. 53.

${ }^{1}$ M. Rousseau, "Floquet wave properties in a periodically layered medium," J. Acoust. Soc. Am. 86, 2369-2376 (1989).

${ }^{2}$ A. M. B. Braga and G. Herrmann, "Wave propagation in submerged layered composites," in Elastic Wave Propagation (Elsevier, Amsterdam, 1989), pp. 301-306.

${ }^{3}$ D. A. Sotiropoulos, "Dispersion of elastic waves in periodically inhomogeneous media," Comput. Mech. 12, 134-146 (1993).

${ }^{4}$ N. A. Day, C. Zhu, and V. K. Kinra, "A study of dispersive waves propagation in periodic layered composites," Rev. Prog. Quant. Nondest. Eval. 13, 243-250 (1994).

${ }^{5}$ R. Esquivel-Sirvent and G. H. Cocoletzi, "Band structure for the propagation of elastic waves in superlattices," J. Acoust. Soc. Am. 95(1), 86-90 (1994).

${ }^{6}$ A. Bedford and D. S. Drumheller, Introduction to Elastic Wave Propagation (Wiley, Chichester, 1994), pp. 1-312.

${ }^{7} \mathrm{~W}$. Cao and W. Qi, "Plane wave propagation in finite 2-2 composites," J. Appl. Phys. 78, 4627-4632 (1995).

${ }^{8}$ F. Kobayashi, S. Biwa, and N. Ohno, "Wave transmission characteristics in periodic media of finite length: Multilayers and fiber arrays," Int. J. Solids Struct. 41, 7361-7375 (2004).

${ }^{9} \mathrm{G}$. Wang, D. Yu, J. Wen, Y. Liu, and X. Wen, "One-dimensional phononic crystals with locally resonant structures," Phys. Lett. 327, 512-521 (2004).

${ }^{10}$ M. I. Hussein, G. M. Hulbert, and R. A. Scott, "Dispersive elastodynamics of 1D banded materials and structures: Analysis," J. Sound Vib. 289, 779-806 (2006).

${ }^{11}$ A. P. Velo, G. A. Gazonas, E. Bruder, and N. Rodriguez, "Recursive dispersion relation in one-dimensional periodic elastic media," SIAM J. Appl. Math. 69(3), 670-689 (2008).

${ }^{12}$ A.-L. Chen and Y.-S. Wang, "Study on band gaps of elastic waves propagating in one-dimensional disordered phononic crystals," Physicia B 392, 369-378 (2007).

${ }^{13}$ M. V. Golub, S. I. Fomenko, T. Q. Bui, C. Zhang, and Y.-S. Wang, "Transmission and band gaps of elastic SH waves in functionally graded periodic laminates,” Int. J. Solids Struct. 49, 344-354 (2012).

${ }^{14}$ Y. Roganov and A. Stovas, "Low-frequency wave propagation in periodically layered media,” Geophys. Prospect. 60, 825-837 (2012). 
${ }^{15}$ B. Djafari-Rouhani, L. Dobrzynski, O. H. Duparc, R. E. Camley, and A. A. Maradudin, "Sagittal elastic waves in infinite and semi-infinite superlattices," Phys. Rev. B 28(4), 1711-1720 (1983).

${ }^{16}$ J. Sapriel and B. D. Rouhani, "Vibrations in superlattices," Surf. Sci. Rep. 10, 189-275 (1989).

${ }^{17}$ Z. Shi, Z. Cheng, and H. Xiang, "Seismic isolation foundations with effective attenuation zones," Soil Dyn. Earthquake Eng. 57, 143-151 (2014).

${ }^{18}$ Y. Yan, Y. L. Mo, F.-Y. Menq, K. H. Stokoe, J. Perkins, and Y. Tang, "Development of seismic isolation systems using periodic materials," Technical Report No. 3219, NEUP (Nuclear Energy University Program) (2014), pp. 1-191.

${ }^{19}$ M. Yamada and S. Nemat-Nasser, "Harmonic waves with arbitrary propagation direction in layered orthotropic elastic composites," J. Compos. Mater. 15, 531-542 (1981).

${ }^{20}$ S. Nemat-Nasser and M. Yamada, "Harmonic waves in layered transversely isotropic composites," J. Sound. Vib. 79(2), 161-170 (1981).

${ }^{21}$ A. H. Nayfeh, "The propagation of horizontally polarized shear waves in multilayered anisotropic media," J. Acoust. Soc. Am. 86(5), 2007-2012 (1989).

${ }^{22}$ T. Lhermitte, B. Perrin, and M. Fink, "Ultrasonic spectroscopy in cross-ply fiber reinforced composites applied to dispersion effects characterization of elastic shear wave," J. Phys. Colloq. 51, C2-1265-C2-1268 (1990).

${ }^{23}$ A. H. Nayfeh, "The general problem of elastic wave propagation in multilayered anisotropic media," J. Acoust. Soc. Am. 89(4), 1521-1531 (1991).

${ }^{24}$ P. J. Shull, D. E. Chimenti, and S. K. Datta, "Elastic guided wave and the Floquet concept in periodically layered plates," J. Acoust. Soc. Am. 95(1), 99-108 (1994).

${ }^{25}$ A. M. B. Braga and G. Herrmann, "Floquet waves in anisotropic periodically layered composites," J. Acoust. Soc. Am. 91(3), 1211-1227 (1992).

${ }^{26}$ A. N. Stroh, "Steady state problems in anisotropic elasticity," J. Math. Phys. 41, 77-103 (1962).

${ }^{27}$ C. Potel, J.-F. Belleval, and Y. Gargouri, "Floquet waves and classical plane waves in an anisotropic periodically multilayered medium: Application to the validity domain of homogenization," J. Acoust. Soc. Am. 97(5), 2815-2825 (1995).

${ }^{28}$ L. Wang and S. I. Rokhlin, "Ultrasonic wave interaction with multidirectional composites: Modeling and experiment," J. Acoust. Soc. Am. 114(5), 2582-2895 (2003).

${ }^{29}$ L. Wang and S. I. Rokhlin, "Floquet wave homogenization of periodic anisotropic media," J. Acoust. Soc. Am. 112(1), 38-45 (2002).

${ }^{30} \mathrm{~L}$. Wang and S. I. Rokhlin, "Floquet wave ultrasonic method for determination of single ply moduli in multidirectional composites," J. Acoust. Soc. Am. 112(3), 916-924 (2002).

${ }^{31}$ M. B. Amor, M. H. B. Ghozlen, and P. Lanceleur, "Frequency-dependent homogenized properties of composite using spectral analysis method," Mater. Sci. Eng. 13, 012033 (2010).

${ }^{32} \mathrm{~N}$. Sela, O. Ishai, and L. Banks-Sills, "The effect of adhesive thickness on interlaminar fracture toughness of interleaved CFRP specimens," Composites 20(3), 257-264 (1989).

${ }^{33}$ S. I. Rokhlin and Y. J. Wang, "Analysis of boundary conditions for elastic wave interaction with an interface between two solids," J. Acoust. Soc. Am. 89(2), 503-515 (1991).

${ }^{34}$ J. P. Jones and J. S. Whittier, "Waves at flexibly bonded interface," J. Appl. Mech. 34, 905-909 (1967).
${ }^{35}$ M. Schoenberg, "Elastic wave behavior across elastic linear slip interfaces," J. Acoust. Soc. Am. 68(5), 1516-1521 (1980).

${ }^{36}$ J.-M. Baik and R. B. Thompson, "Ultrasonic scattering from imperfect interfaces: A quasi-static model," J. Nondestruct. Eval. 4, 177-196 (1984).

${ }^{37}$ A. Pilarski and J. L. Rose, "A transverse-wave ultrasonic obliqueincidence technique for interfacial weakness detection in adhesive bonds," J. Appl. Phys. 63(2), 300-307 (1988).

${ }^{38} \mathrm{~A}$. Boström and G. Wickham, "On the boundary conditions for ultrasonic transmission by partially closed cracks," J. Nondestruct. Eval. 10(4), 139-149 (1991).

${ }^{39}$ S. I. Rokhlin and W. Huang, "Ultrasonic wave interaction with a thin anisotropic layer between two anisotropic solids: Exact and asymptoticboundary-condition methods," J. Acoust. Soc. Am. 92(3), 1729-1742 (1992).

${ }^{40} \mathrm{P}$. Fraisse, F. Schmit, and A. Zarembowitch, "Ultrasonic inspection of very thin adhesive layers,” J. Appl. Phys. 72(8), 3264-3271 (1992).

${ }^{41}$ K. Heller, L. J. Jacobs, and J. Qu, "Characterization of adhesive bond properties using Lamb waves," NDT\&E Int. 33, 555-563 (2000).

${ }^{42}$ M. V. Golub, "Propagation of elastic waves in layered composites with microdefect concentration zones and their simulation with spring boundary conditions," Acoust. Phys. 56(6), 848-855 (2010).

${ }^{43} \mathrm{M}$. V. Golub and A. Boström, "Spring boundary conditions and modeling of 2D wave propagation in composites with imperfect interfaces," in Proceedings of International Conference Days on Diffraction (2010), pp. 73-78.

${ }^{44}$ Y. Lu and J. D. Achenbach, "Effects of random deviations in interface properties on the propagation of ultrasound in thick composites," J. Acoust. Soc. Am. 90(5), 2576-2585 (1991).

${ }^{45} \mathrm{Y}$. Lu, "Effects of random fluctuations in ply mechanical properties on ultrasound propagation in a laminated solid layer," Ultrasonics 30(5), 289-295 (1992).

${ }^{46}$ Y. Ishii and S. Biwa, "Ultrasonic evaluation of interlayer interfacial stiffness of multilayered structures," J. Appl. Phys. 111, 084907 (2012).

${ }^{47}$ Y. Ishii and S. Biwa, "Evaluation of interlayer interfacial stiffness and layer wave velocity of multilayered structures by ultrasonic spectroscopy," J. Acoust. Soc. Am. 136(1), 183-191 (2014).

${ }^{48}$ Y. Ishii and S. Biwa, "Transmission of ultrasonic waves at oblique incidence to composite laminates with spring-type interlayer interfaces," J. Acoust. Soc. Am. 138(5), 2800-2810 (2015).

${ }^{49}$ L. Wang and S. I. Rokhlin, "Stable reformulation of transfer matrix method for wave propagation in layered anisotropic media," Ultrasonics 39, 413-424 (2001).

${ }^{50}$ S. I. Rokhlin and L. Wang, "Stable recursive algorithm for elastic wave propagation in layered anisotropic media: Stiffness matrix method," J. Acoust. Soc. Am. 112(3), 822-834 (2002).

${ }^{51}$ S. Biwa, Y. Watanabe, and N. Ohno, "Analysis of wave attenuation in unidirectional viscoelastic composites by a differential scheme," Compos. Sci. Technol. 63, 237-247 (2003).

${ }^{52}$ B. A. Auld, Acoustic Fields and Waves in Solids Volume I (Krieger, Malabar, FL, 1990), pp. 135-161.

${ }^{53}$ C. Potel, P. Gatignol, and J.-F. Belleval, "Energetic criterion for the radiation of Floquet waves in infinite anisotropic periodically multilayered media," Acta Acust. Acust. 87(3), 340-351 (2001).

${ }^{54}$ T. Pialucha and P. Cawley, "An investigation of the accuracy of oblique incidence ultrasonic reflection coefficient measurements," J. Acoust. Soc. Am. 96(3), 1651-1660 (1994). 